\title{
Supporting Information Sustainable Process Intensification using Building Blocks
}

\author{
Salih Emre Demirel, Jianping Li, Mahmoud El-Halwagi, and M. M. Faruque Hasan* \\ Artie McFerrin Department of Chemical Engineering, Texas A $3 M$ University \\ College Station, TX 77843-3122, USA
}

*corresponding author: hasan@tamu.edu

Number of Figures: 4

Number of Tables: 6

Number of Pages: 29 
Here we provide some more details on the optimization models and the optimal results. Specifically, we first provide a detailed description of the optimization model summarized in the manuscript along with the simultaneous heat integration model. Then, we provide details on the physical parameters, economic objective functions and building block representations for the base case designs. We also provide more information on the modeling assumptions and design bounds. Then, we present more details on the single objective optimization results and present an analysis of the pareto solutions for the base-case designs. Finally, we provide a detailed cost breakdown for all the presented solutions.

\section{Nomenclature}

\section{Indices}

$\begin{array}{ll}i \in\{1, \ldots,|I|\} & \text { Position of block in the horizontal direction } \\ j \in\{1, \ldots,|J|\} & \text { Position of block in the vertical direction } \\ k \in\{1, \ldots,|K|\} & \text { Chemical components } \\ f s \in\{1, \ldots,|F S|\} & \text { Fresh feed streams } \\ p s \in\{1, \ldots,|P S|\} & \text { Product streams } \\ r \in\{1, \ldots,|R X|\} & \text { Reactions } \\ s \in\{1, \ldots,|S|\} & \text { Separation phenomenon } \\ m \in\{1, \ldots,|M|\} & \text { Separation material }\end{array}$

\section{Sets}

I

$J$

K

$S$

M

$F S$

$P S$

$R X$

$K^{\text {reactants }}(k)$

$\operatorname{Equil}(s, m)$
Set of all rows within the superstructure

Set of all columns within the superstructure

Set of all chemical components

Set of separation phenomenon

Set of separation material

Set of feed streams

Set of product streams

Set of reactions

Set of all reactants

Set of equilibrium-based separation phenomenon s and its related separation phenomenon material $\mathrm{m}$ 


$$
\begin{aligned}
& L N\left(i, j, i^{\prime}, j^{\prime}\right) \\
& S B(i, j) \\
& \text { TB }(i, j) \\
& A B(i, j) \\
& \operatorname{RxnB}(i, j) \\
& \operatorname{Act} F(i, j) \\
& \operatorname{Act} R(i, j) \\
& F e e d B(i, j, f s) \\
& \operatorname{ProdB}(i, j, p s) \\
& k p(k, p s) \\
& H P(i, j) \\
& H N(i, j) \\
& V P(i, j) \\
& V N(i, j) \\
& \operatorname{VLPC}(i, j) \\
& V B(i, j) \\
& L B(i, j) \\
& V B^{\text {check }}(i, j) \\
& \operatorname{LB}^{\text {check }}(i, j)
\end{aligned}
$$

\section{Continuous Variables}

$F_{i, j, k}$

$R_{i, j, k}$

$G_{i, j, k}$

$E F_{i, j}$
Set of jump connections allowed between block $B_{i, j}$ and $B_{i^{\prime}, j^{\prime}}$ Set of blocks $B_{i, j}$ where individual material balances are activated Set of horizontal block pairs $B_{i, j}$ and $B_{i, j+1}$ in which combined material balances are activated (only the block on the left is defined) Set denoting the active blocks, i.e. allowed to have inlet and/or outlet flows

Set of blocks $B_{i, j}$ in which reactions are allowed

Set denoting the active horizontal $F_{i, j, k}$ streams

Set denoting the active vertical $R_{i, j, k}$ streams

Set denoting blocks $B_{i, j}$ where feed $f s$ can be introduced

Set denoting blocks $B_{i, j}$ where product $p s$ can be withdrawn

Set denoting component $k$ with purity restriction in product stream ps

Set denoting the active horizontal positive stream $F P_{i, j, k}$ streams Set denoting the active horizontal negative stream $F N_{i, j, k}$ streams Set denoting the active vertical positive stream $R P_{i, j, k}$ streams Set denoting the active vertical negative stream $R N_{i, j, k}$ streams Set denoting the horizontal pair of blocks separated by vapor liquid phase contact boundary

Set denoting the position of vapor blocks

Set denoting the position of liquid blocks

Set denoting the position of vapor blocks where dew point constraints are active

Set denoting the position of liquid blocks where bubble point constraints are active

Flow rate of component $k$ between block $B_{i, j}$ and block $B_{i, j+1}$ Flow rate of component $k$ between block $B_{i, j}$ and block $B_{i+1, j}$ Generation/Consumption of component $\mathrm{k}$ in block $B_{i, j}$ through all reactions

Enthalpy carried by horizontal stream 
$E R_{i, j}$

$E M_{i, j}$

$E P_{i, j}$

$E J_{i, j}^{p}$

$E J_{i, j}^{f}$

React $_{i, j, k, r}$

\section{Positive Variables}

$T_{i, j}$

$P_{i, j}$

$F P_{i, j, k}$

$F N_{i, j, k}$

$R P_{i, j, k}$

$R N_{i, j, k}$

$J_{i, j, i^{\prime}, j^{\prime}, k}$

$M_{i, j, k, f s}$

$P_{i, j, k, p s}$

$z_{i, j, f s}^{f e e d f r a c}$

$y_{i, j, k}$

$C_{i, j, k}$

$\rho_{i, j, k}$

$F P_{i, j}^{T}$

$F N_{i, j}^{T}$

$R P_{i, j}^{T}$

$R N_{i, j}^{T}$

$J_{i, j, i^{\prime}, j^{\prime}}^{T}$

$P_{i, j, p s}^{p, t o t a l}$
Enthalpy carried by vertical stream

Enthalpy introduced by feed streams

Enthalpy carried out from $B_{i, j}$ by product streams

Enthalpy introduced into $B_{i, j}$ by jump streams

Enthalpy carried out from $B_{i, j}$ by jump streams

Molar consumption/generation of component $k$ via reaction $r$ in block $B_{i, j}$

Temperature of block $B_{i, j}$

Pressure of block $B_{i, j}$

Flow rate of the positive (left to right) part of $F_{i, j, k}$

Flow rate of the negative (right to left) part of $F_{i, j, k}$

Flow rate of the positive (downwards) part of $R_{i, j, k}$

Flow rate of the negative (upwards) part of $R_{i, j, k}$

Flow rate of the component $k$ in jump stream between $B_{i, j}$ and $B_{i^{\prime}, j^{\prime}}$

Flow rate of component $k$ in feed stream $f s$ introduced into block $B_{i, j}$

Flow rate of component $k$ in product stream $p s$ withdrawn from block $B_{i, j}$

Fraction of the feed stream $f s$ introduced into block $B_{i, j}$

Mole fraction of $k$ in block $B_{i, j}$

Molar composition of $k$ in the mixture in block $B_{i, j}$

Pure component molar composition of $k$ in block $B_{i, j}$

Total flow rate of the positive (left to right) part of $F_{i, j, k}$

Total flow rate of the negative (right to left) part of $F_{i, j, k}$

Total flow rate of the positive (downwards) part of $R_{i, j, k}$

Total flow rate of the negative (upwards) part of $R_{i, j, k}$

Total flow rate of the jump stream between $B_{i, j}$ and $B_{i^{\prime}, j^{\prime}}$

Total flow rate of the product stream $p s$ withdrawn from $B_{i, j}$ 
Cons $_{i, j, r}$

$V_{i, j}$

$K_{i, j, k, s, m}^{e q}$

$P_{i, j, k}^{s a t}$

$P_{i, j}^{b u b}$

$P_{i, j}^{\text {dew }}$

$Q_{i, j}^{h}$

$Q_{i, j}^{c}$

$Q_{i, j}^{h 2}$

$Q_{i, j}^{c 2}$

$J_{i, j, i^{\prime}, j^{\prime}}^{p u m p}$

$\Delta P_{i, j}$

\section{Negative Variables}

$E F P_{i, j, k}^{l}$

EFP $P_{i, j, k}^{g}$

$E F N_{i, j, k}^{l}$

$E F N_{i, j, k}^{g}$

$E R P_{i, j, k}^{l}$

$E R P_{i, j, k}^{g}$

$E R N_{i, j, k}^{l}$

$E R N_{i, j, k}^{g}$

\section{Binary Variables}

$z_{i, j}^{c r R}$

\section{Parameters}

$F_{f s}^{\text {feed }}$
The consumption of key component in reaction $r$ in block $B_{i, j}$ per volume

Reaction volume in block $B_{i, j}$

Equilibrium constant of component $k$ for separation phenomena $s$ with enabling material $m$ in block $B_{i, j}$

Saturation pressure for component $k$ in block $B_{i, j}$

Bubble pressure of block $B_{i, j}$

Dew pressure of block $B_{i, j}$

Heat introduced into $B_{i, j}$ by hot utility

Heat withdrawn from $B_{i, j}$ by cold utility

Heat introduced into horizontal block pair $B_{i, j}$ and $B_{i, j+1}$ by hot utility

Heat withdrawn from horizontal block pair $B_{i, j}$ and $B_{i, j+1}$ by cold utility

Work required for the pump on the jump stream between $B_{i, j}$ and $B_{i^{\prime}, j^{\prime}}$

Pressure drop between $B_{i, j}$ and $B_{i+1, j}$

Enthalpy of component $k$ in liquid stream $F P_{i, j, k}$

Enthalpy of component $k$ in vapor stream $F P_{i, j, k}$

Enthalpy of component $k$ in liquid stream $F N_{i, j, k}$

Enthalpy of component $k$ in vapor stream $F N_{i, j, k}$

Enthalpy of component $k$ in liquid stream $R P_{i, j, k}$

Enthalpy of component $k$ in vapor stream $R P_{i, j, k}$

Enthalpy of component $k$ in liquid stream $R N_{i, j, k}$

Enthalpy of component $k$ in vapor stream $R N_{i, j, k}$

Completely restricted boundary between vertical blocks $B_{i, j}$ and $B_{i+1, j}$

Total flow rate of feed stream $f s$ 


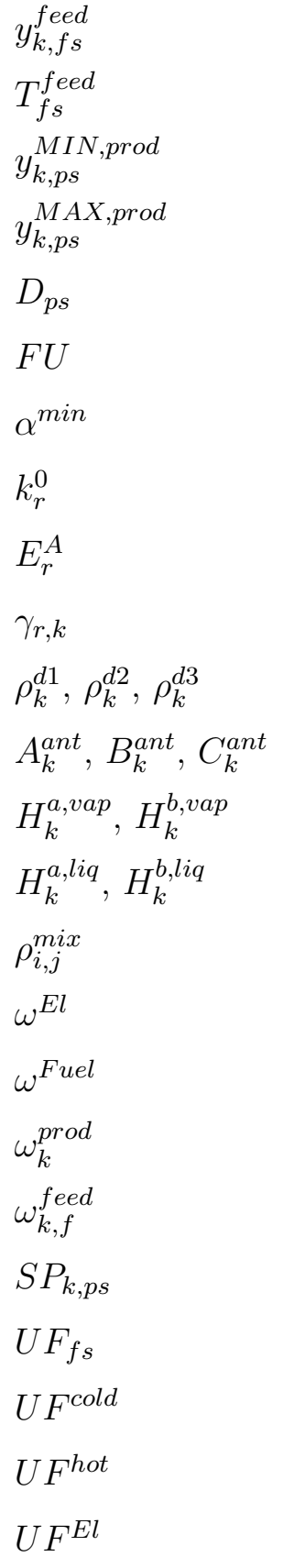

Mole fraction of component $k$ in feed stream $f s$

Temperature of feed stream $f s$

Minimum purity of component $k$ in product stream $p s$

Maximum purity of component $k$ in product stream $p s$

Demand for product stream ps

Upper bound on flow rates

Minimum flow parameter $\left(10^{-6}\right)$

Pre-exponential factor for reaction $r$

Activation energy for reaction $r$

Stoichiometric coefficient of $k$ in reaction $r$

Pure liquid molar density coefficients for $k$

Antoine coefficients for component $k$

Vapor enthalpy coefficients for component $k$

Liquid enthalpy coefficients for component $k$

Density of fluid mixture for the pump work calculation

$\mathrm{CO}_{2}$-eq emissions from electricity consumption

$\mathrm{CO}_{2}$-eq emissions from hot utility consumption

$\mathrm{CO}_{2}$-eq emissions from the product streams

$\mathrm{CO}_{2}$-eq emissions from fresh raw material consumption

Selling price for $k$ in product stream $p s$

Unit price of feed stream $f s$

Unit price of cold utility stream

Unit price of hot utility stream

Unit price of electricity

\section{Optimization Model}

The general mathematical model equations given in the manuscript are explained in detail here. We also provide more details on the constraints given $g(x) \leq 0$ and how are these implemented for the presented case study. Here, equation numbers without ' $S$ ' refers to the equations within the manuscript.

Equations 1 and 2 serve as the material balance constraints for standalone and vapor-liquid 
phase contact block pairs, respectively. Note that one block can only belong to either $S B$ or $T B$ set. Using $T B$ set eliminates the need for interblock variables when an equilibrium-based separation model is adopted as it is the case in vapor-liquid phase contact blocks. $G_{i, j, k}$ term in Eq. 3 describes the generation terms due to reactions. This is implemented for the ethylene glycol production as follows:

$$
\begin{gathered}
G_{i, j, k}=\sum_{r \in R X N} \operatorname{React}_{i, j, k, r} \quad i, j \in R x n B, k \in K \\
\text { React }_{i, j, k, r}=\text { Cons }_{i, j, r} \gamma_{r, k} V_{i, j} \quad i, j \in R x n B, \gamma_{r, k} \neq 0 \\
\text { Cons }_{i, j, r}=f^{r x n}\left(T_{i, j}, C_{i, j, k}, k_{r}^{0}, E_{r}^{A}\right) \quad i, j \in R x n B, r \in R x n
\end{gathered}
$$

Here, the reaction set includes $r=\{$ main, side $\}$ and reaction rates in Cons $_{i, j, r}$ term denotes the reaction rate for reaction $r$ per unit volume and the reaction rate is calculated based on block reaction volume, $V_{i, j}$, with Eq. S2. Set $\operatorname{Rxn} B$ determines the building blocks $B_{i, j}$ at which reaction is allowed. Since the reaction is homogeneous liquid phase reactions, these blocks are in liquid phase. Equation S3 is implemented through following homogeneous kinetic rate expressions obtained from Altıokka and Karayalçın (2009): ${ }^{1}$

$$
\begin{aligned}
& -r_{E O}^{\text {main }}[\mathrm{kmol} / \mathrm{h}]=60 \times \exp \left(13.62-\left(\frac{8220}{T[K]}\right)\right) C_{E O}\left[\mathrm{kmol} / \mathrm{m}^{3}\right] \times C_{W}\left[\mathrm{kmol} / \mathrm{m}^{3}\right] \\
& -r_{E O}^{\text {side }}[\mathrm{kmol} / \mathrm{h}]=60 \times \exp \left(15.57-\left(\frac{8700}{T[K]}\right)\right) C_{E O}\left[\mathrm{kmol} / \mathrm{m}^{3}\right] \times C_{E G}\left[\mathrm{kmol} / \mathrm{m}^{3}\right]
\end{aligned}
$$

Here, reactant concentrations are in molar concentration. These molar concentrations are calculated based on molar fractions and assuming a linear mixing rule which requires pure molar concentration of each species. Accordingly, following constitutive equations are used:

$$
\begin{gathered}
C_{i, j, k} \times\left(\sum_{k^{\prime} \in K} \frac{y_{i, j, k^{\prime}}}{\rho_{i, j, k^{\prime}}}\right)=y_{i, j, k} \quad i, j \in R x n B, k \in K^{\text {reactants }} \\
\rho_{i, j, k}=\rho_{k}^{d 1}+\rho_{k}^{d 2} \times T_{i, j}+\rho_{k}^{d 3} \times T_{i, j}^{2} \quad i, j \in \operatorname{Rxn} B, k \in K
\end{gathered}
$$

$K^{\text {reactants }}$ denotes the set of components $k$ that act as reactants in the system, i.e. EO, EG and W. Here, $\rho_{i, j, k}$, molar concentration of pure components are obtained from ASPEN Plus 
and fitted to the polynomial expression given in Eq. S7 as a function of temperature. These parameters are given below in physical parameters section. With these, extent of reaction terms become:

$$
\begin{aligned}
& \text { Cons }_{i, j, r=\text { Main }}=60 \times \exp \left(13.62-\left(\frac{8220}{T_{i, j}}\right)\right) C_{i, j, k=E O} \times C_{i, j, k=W}, \quad i, j \in R x n B \\
& \text { Cons }_{i, j, r=\text { Side }}=60 \times \exp \left(15.57-\left(\frac{8700}{T_{i, j}}\right)\right) C_{i, j, k=E O} \times C_{i, j, k=E G}, \quad i, j \in R x n B
\end{aligned}
$$

Fresh raw materials $f s$ can be introduced into any block included in set FeedB. As the feed streams are in liquid phase, these blocks also are in liquid phase. The feed availability and compositions are ensured via the following:

$$
\begin{gathered}
M_{i, j, k, f s}=F_{f s}^{f e e d} y_{k, f s}^{\text {feed }} z_{i, j, f s}^{\text {feedfrac }}, \quad i, j, f s \in \text { FeedB } \\
\sum_{i, j, f s \in \text { FeedB }} z_{i, j, f s}^{\text {feedfrac }} \leq 1 \\
z_{i, j, f s}^{\text {feedfrac }} \leq 1, \quad i, j, f s \in \text { FeedB }
\end{gathered}
$$

Here, $z_{i, j, f s}^{\text {feedfrac }}$ is a $0-1$ continuous variable denoting what fraction of the total available feed stream is introduced into the block $B_{i, j}$.

Equations 4 denote the horizontal and vertical flow rates with respect to their directions. $F_{i, j, k}$ and $R_{i, j, k}$ denote the flow rate through a horizontal and vertical boundary, respectively. These terms are activated according to the sets $A c t F$ and $A c t R$. Each flow within the superstructure is allowed to flow in both directions. If the horizontal flow is in positive (negative) direction, then $F P_{i, j, k}\left(F N_{i, j, k}\right)$ is active. Similarly, vertical flow $R_{i, j, k}$ is also comprised of two counterparts as $R P_{i, j, k}$ and $R N_{i, j, k}$. Only one of these counterparts can be active at one time. In the original model presented in Demirel et al., ${ }^{2}$ this is ensured through binary variables. Here, we pre-specify the directions with the subsets. While $H P$ and $H N$ denote the active horizontal positive and negative flow components, respectively, $V P$ and $V N$ denote the vertical positive and negative flow components, respectively.

Equations 5-6 are for satisfying product purity and demand. Stream splitting through unrestricted boundaries, jump flows and product streams are satisfied through Eqns. 7-9. Here, 
total molar flow rates are used which are defined as follows:

$$
\begin{gathered}
F P_{i, j}^{T}=\sum_{k \in K} F P_{i, j, k} ; \quad F N_{i, j}^{T}=\sum_{k \in K} F N_{i, j, k} \quad i, j \in A c t F \\
R P_{i, j}^{T}=\sum_{k \in K} R P_{i, j, k} ; \quad R N_{i, j}^{T}=\sum_{k \in K} R N_{i, j, k} \quad i, j \in \operatorname{Act} R \\
J_{i, j, i^{\prime}, j^{\prime}}^{T}=\sum_{k \in K} J_{i, j, i^{\prime}, j^{\prime} k} \quad i, j, i^{\prime}, j^{\prime} \in L N \\
P_{i, j, p s}^{p, t o t a l}=\sum_{k \in K} P_{i, j, k, p s} \quad i, j, p s \in \operatorname{Prod} B \\
\sum_{k \in K} y_{i, j, k}=1 \quad i, j \in A B
\end{gathered}
$$

Equation 10 is to satisfy the thermodynamic equilibrium between the horizontal building block pairs when the block on the left is in liquid and the block on the right is in vapor phase. The position of these phases can be also reversed and also the orientation can be in vertical direction. These type of more general equations can be found in Demirel et al. (2017). ${ }^{2}$ Here, we only consider horizontal direction and assign liquid phase to the block on the left. $V L P C$ set designates the blocks $B_{i, j}$ in which these phase calculations are valid. Set indices $s$ and $m$ refers to the separation phenomena and enabling material sets, respectively. In this work, we only consider vapor-liquid phase contact phenomena for the separation which does not require any enabling material, e.g. membrane, absorbent. Accordingly, $s=\{V L-P C\}$ and $m=\{$ null $\}$. Equil set designates an equilibrium-based separation phenomena and material. These sets might not be needed within the scope of the presented case study as we consider single type of phenomena, yet we include them for the consistency with the original model. In Equation $10, K_{i, j, k, s, m}^{e q}$ denotes the phase equilibrium constant which can depend on the block compositions, temperatures and pressures. Here, we assume ideal phase equilibrium. Then, following relations are written:

$$
\begin{gathered}
K_{i, j, k, s, m}^{e q} P_{i, j}=P_{i, j, k}^{s a t} \quad i, j \in V L P C, k \in K, s, m \in \text { Equil } \\
P_{i, j, k}^{s a t}=\exp \left(A_{k}^{a n t}-\frac{B_{k}^{a n t}}{T_{i, j}+C_{k}^{a n t}}\right) \quad i, j \in V L P C \cup L B^{\text {check }}, k \in K
\end{gathered}
$$

Here, Eq. $\mathrm{S} 19$ is to calculate the saturation pressure based on Antoine equation. Antoine parameters are obtained from ASPEN Plus and given in the next section. Note that although 
equilibrium calculations are performed for each block within the $V L P C$ set, Antoine equation is also valid for blocks where phase of the blocks are checked for feasible operation. For instance, condensers for the base case designs are assumed to be total condensers and phase of these blocks are ensured to be in liquid phase with Eqs. 11. Specifically, these constraints are written as follows:

$$
\begin{gathered}
P_{i, j} \geq \sum_{k \in K} y_{i, j, k} P_{i, j, k}^{s a t} i, j \in L B^{\text {check }} \\
P_{i, j} \leq \frac{1}{\sum_{k \in K} \frac{y_{i, j, k}}{P_{i, j, k}^{s a t}}} i, j \in V B^{\text {check }}
\end{gathered}
$$

while Eq. S20 checks for bubble pressure, Eq. S21 checks for dew pressure. Sets $L B^{\text {check }}$ and $V B^{\text {check }}$ designate the block positions where these equations are valid.

Equation 12 is to calculate the pump work required for increasing the pressure of a liquid stream for jump streams. We only allowed for liquid pressure increase through these jump streams within this work. Similar constraints can be written for other connections and also vapor streams for vapor compression work as given in the original model. ${ }^{3}$ Yet, in this work, we did not utilize vapor compression.

Equations 13-14 describe the energy balances. While the former is used for single blocks, the latter is used for two block energy balances for the vapor-liquid phase contact block pairs. Similar to the material balance equations, defining overall energy balance for the horizontal pairs decreases the model complexity. Enthalpy terms in these equations are implemented as follows. All these terms are dissected into several parts based on phase and the direction of the flows. For horizontal direction, for example, $E F_{i, j}$ is dissected into four terms based on the direction and the phase as follows:

$$
E F_{i, j}=-\sum_{k \in K} E F P_{i, j, k}^{g}-\sum_{k \in K} E F P_{i, j, k}^{l}+\sum_{k \in K} E F N_{i, j, k}^{g}+\sum_{k \in K} E F N_{i, j, k}^{l}, \quad i, j \in A c t F
$$

Similarly, $E R_{i, j}$ is dissected into four terms as follows:

$$
E R_{i, j}=-\sum_{k \in K} E R P_{i, j, k}^{g}-\sum_{k \in K} E R P_{i, j, k}^{l}+\sum_{k \in K} E R N_{i, j, k}^{g}+\sum_{k \in K} E R N_{i, j, k}^{l}, \quad i, j \in A c t R
$$

These individual stream enthalpy terms are defined as follows as a function of temperature (based 
on ideal gas assumption):

$$
\begin{aligned}
& E F P_{i, j, k}^{l}=F P_{i, j, k}\left(H_{k}^{a, l i q}+H_{k}^{b, l i q} T_{i, j}\right) \quad i, j \in H P^{l i q}, k \in K \\
& E F P_{i, j, k}^{g}=F P_{i, j, k}\left(H_{k}^{a, v a p}+H_{k}^{b, v a p} T_{i, j}\right) \quad i, j \in H P^{v a p}, k \in K \\
& E F N_{i, j, k}^{l}=F N_{i, j, k}\left(H_{k}^{a, l i q}+H_{k}^{b, l i q} T_{i, j+1}\right) \quad i, j \in H N^{l i q}, k \in K \\
& E F N_{i, j, k}^{g}=F N_{i, j, k}\left(H_{k}^{a, v a p}+H_{k}^{b, v a p} T_{i, j+1}\right) \quad i, j \in H N^{v a p}, k \in K \\
& E R P_{i, j, k}^{l}=R P_{i, j, k}\left(H_{k}^{a, l i q}+H_{k}^{b, l i q} T_{i, j}\right) \quad i, j \in V P^{l i q}, k \in K \\
& E R P_{i, j, k}^{g}=R P_{i, j, k}\left(H_{k}^{a, v a p}+H_{k}^{b, v a p} T_{i, j}\right) \quad i, j \in V P^{v a p}, k \in K \\
& E R N_{i, j, k}^{l}=R N_{i, j, k}\left(H_{k}^{a, l i q}+H_{k}^{b, l i q} T_{i+1, j}\right) \quad i, j \in V N^{l i q}, k \in K \\
& E R N_{i, j, k}^{g}=R N_{i, j, k}\left(H_{k}^{a, v a p}+H_{k}^{b, v a p} T_{i+1, j}\right) \quad i, j \in V N^{v a p}, k \in K
\end{aligned}
$$

Here, subsets are used to designate the position of the interblock streams for which these constraints active. For instance, $H P^{l i q}$ is to denote the active $F P_{i, j, k}$ streams for which the enthalpy is calculated based on Eq. S24. Note that $H P^{l i q} \subseteq H P$ which states that even a horizontal positive stream exists, enthalpy calculations might not be needed for this stream. This is allowed to simplify the model if a block is only used for material transfer with single inlet and outlet not requiring any energy balance calculations.

Similar enthalpy relations are also written for the jump streams, external feed and product streams as below:

$$
\begin{gathered}
E J_{i, j, i^{\prime}, j^{\prime}, k}^{l}=J_{i, j, i^{\prime}, j^{\prime}, k}\left(H_{k}^{a, l i q}+H_{k}^{b, l i q} T_{i, j}\right) \quad i, j, i^{\prime}, j^{\prime} \in L N^{l i q}, k \in K \\
E J_{i, j, i^{\prime}, j^{\prime}, k}^{g}=J_{i, j, i^{\prime}, j^{\prime}, k}\left(H_{k}^{a, v a p}+H_{k}^{b, v a p} T_{i, j}\right) \quad i, j, i^{\prime}, j^{\prime} \in L N^{v a p}, k \in K \\
E M_{i, j}=\sum_{k \in K} \sum_{f s \in F S} M_{i, j, k, f s}\left(H_{k}^{a, l i q}+H_{k}^{b, l i q} T_{f s}^{f e e d}\right) \quad i, j \in F B \\
E P_{i, j}=\sum_{k \in K} \sum_{p \in P S} P_{i, j, k, p s}\left(H_{k}^{a, l i q}+H_{k}^{b, l i q} T_{i, j}\right) \quad i, j \in P B
\end{gathered}
$$

By using these enthalpy terms, energy balance constraints Eq. 13 for the single block energy 
balance can be rewritten as follows:

$$
\begin{aligned}
& \sum_{k \in K} E F P_{i, j-1, k}^{g}+\sum_{k \in K} E F P_{i, j-1, k}^{l}-\sum_{k \in K} E F N_{i, j-1, k}^{g}-\sum_{k \in K} E F N_{i, j-1, k}^{l}+\sum_{k \in K} E R P_{i-1, j, k}^{g} \\
& +\sum_{k \in K} E R P_{i-1, j, k}^{l}-\sum_{k \in K} E R N_{i-1, j, k}^{g}-\sum_{k \in K} E R N_{i-1, j, k}^{l}-\sum_{k \in K} E F P_{i, j, k}^{g}-\sum_{k \in K} E F P_{i, j, k}^{l} \\
& +\sum_{k \in K} E F N_{i, j, k}^{g}+\sum_{k \in K} E F N_{i, j, k}^{l}-\sum_{k \in K} E R P_{i, j, k}^{g}-\sum_{k \in K} E R P_{i, j, k}^{l}+\sum_{k \in K} E R N_{i, j, k}^{g} \\
& +\sum_{k \in K} E R N_{i, j, k}^{l}+\sum_{i^{\prime}, j^{\prime} \in L N} \sum_{k \in K} E J_{i^{\prime}, j^{\prime}, i, j, k}^{g}+\sum_{i^{\prime}, j^{\prime} \in L N} \sum_{k \in K} E J_{i^{\prime}, j^{\prime}, i, j, k}^{l}-\sum_{i^{\prime}, j^{\prime} \in L N} \sum_{k \in K} E J_{i, j, i^{\prime}, j^{\prime}, k}^{g} \\
& -\sum_{i^{\prime}, j^{\prime} \in L N} \sum_{k \in K} E J_{i, j, i^{\prime}, j^{\prime}, k}^{l}+E M_{i, j}-E P_{i, j}+Q_{i, j}^{h}-Q_{i, j}^{c}=0, \quad i, j \in S B
\end{aligned}
$$

Similarly, two block energy balance equation Eq. 14. can be rewritten as follows:

$$
\begin{aligned}
& \sum_{k \in K} E F P_{i, j-1, k}^{g}+\sum_{k \in K} E F P_{i, j-1, k}^{l}-\sum_{k \in K} E F N_{i, j-1, k}^{g}-\sum_{k \in K} E F N_{i, j-1, k}^{l}+\sum_{k \in K} E R P_{i-1, j, k}^{g} \\
& +\sum_{k \in K} E R P_{i-1, j, k}^{l}-\sum_{k \in K} E R N_{i-1, j, k}^{g}-\sum_{k \in K} E R N_{i-1, j, k}^{l}-\sum_{k \in K} E F P_{i, j+1, k}^{g}-\sum_{k \in K} E F P_{i, j+1, k}^{l} \\
& +\sum_{k \in K} E F N_{i, j+1, k}^{g}+\sum_{k \in K} E F N_{i, j+1, k}^{l}-\sum_{k \in K} E R P_{i, j, k}^{g}-\sum_{k \in K} E R P_{i, j, k}^{l}+\sum_{k \in K} E R N_{i, j, k}^{g} \\
& +\sum_{k \in K} E R N_{i, j, k}^{l}-\sum_{k \in K} E R P_{i, j+1, k}^{g}-\sum_{k \in K} E R P_{i, j+1, k}^{l}+\sum_{k \in K} E R N_{i, j+1, k}^{g}+\sum_{k \in K} E R N_{i, j+1, k}^{l} \\
& +\sum_{k \in K} E R P_{i-1, j+1, k}^{g}+\sum_{k \in K} E R P_{i-1, j+1, k}^{l}-\sum_{k \in K} E R N_{i-1, j+1, k}^{g}-\sum_{k \in K} E R N_{i-1, j+1, k}^{l} \\
& +\sum_{i^{\prime}, j^{\prime} \in L N} \sum_{k \in K} E J_{i^{\prime}, j^{\prime}, i, j, k}^{g}+\sum_{i^{\prime}, j^{\prime} \in L N} \sum_{k \in K} E J_{i^{\prime}, j^{\prime}, i, j, k}^{l}-\sum_{i^{\prime}, j^{\prime} \in L N} \sum_{k \in K} E J_{i, j, i^{\prime}, j^{\prime}, k}^{g} \\
& -\sum_{i^{\prime}, j^{\prime} \in L N} \sum_{k \in K} E J_{i, j, i^{\prime}, j^{\prime}, k}^{l}+\sum_{i^{\prime}, j^{\prime} \in L N} \sum_{k \in K} E J_{i^{\prime}, j^{\prime}, i, j+1, k}^{g}+\sum_{i^{\prime}, j^{\prime} \in L N} \sum_{k \in K} E J_{i^{\prime}, j^{\prime}, i, j+1, k}^{l} \\
& -\sum_{i^{\prime}, j^{\prime} \in L N} \sum_{k \in K} E J_{i, j+1, i^{\prime}, j^{\prime}, k}^{g}-\sum_{i^{\prime}, j^{\prime} \in L N} \sum_{k \in K} E J_{i, j+1, i^{\prime}, j^{\prime}, k}^{l}+E M_{i, j}-E P_{i, j}+E M_{i, j+1} \\
& -E P_{i, j+1}+Q_{i, j}^{h 2}-Q_{i, j}^{c 2}=0, \sum_{i, j} \in B_{i}
\end{aligned}
$$

Here, $Q_{i, j}^{h 2} Q_{i, j}^{c 2}$ are the heat duty variables for the two neighboring blocks. The relationship between these variables and the block heat duty variables is as follow:

$$
Q_{i, j}^{h 2}-Q_{i, j}^{c 2}=Q_{i, j}^{h}-Q_{i, j}^{c}+Q_{i, j+1}^{h}-Q_{i, j+1}^{c}, \quad i, j \in T B
$$

As mentioned earlier, a block can belong to either $S B$ or $T B$ set. Hence, when a block belongs to $T B$, single block heat duty variables are not utilized and fixed to be zero.

Some variables although appear in material and energy balances, may not be necessary. 
These variables are also fixed to be zero. For instance, if an interblock horizontal stream flows in positive direction, i.e. $F P_{i, j, k}$ is active, and belongs to $H P$ set, then the negative counterpart is fixed to be zero, i.e. $F N_{i, j, k}=0$. Similarly, all the associated energy balance variables are taken as zero. Similarly, for the reaction variables, we write the following:

$$
\begin{aligned}
& G_{i, j, k}=0, \quad i, j \notin R x n B, k \in K \\
& \text { Cons }_{i, j, r}=0, \quad i, j \notin R x n B, r \in R x n \\
& \operatorname{React}_{i, j, k, r}=0, i, j \notin R x n B, \gamma_{r, k}=0, k \in K
\end{aligned}
$$

These result in significant reduction in the model size.

\section{Simultaneous Heat Exchanger Network Synthesis Model}

The idea behind the simultaneous heat integration model is to allow the heat duty variables at different locations within the superstructure to be matched with each other. There are two different types of heat duty variables: (i) block heat duty variables, i.e. $Q_{i, j}^{h}$ and $Q_{i, j}^{c}$, and (ii) heat duty variables for the horizontal building block pairs, i.e. $Q_{i, j}^{h 2}$ and $Q_{i, j}^{c 2}$. All these variables can be allowed to match with each other ad four different type of heat exchange schemes can be considered. In the presented case study, we considered simultaneous heat integration between condenser and reboiler blocks. While total condenser blocks include only cooling heat duty terms, i.e. $Q_{i, j}^{c}$, reboiler blocks, which are taken as partial reboilers, include only heating duty for horizontal block pairs, i.e. $Q_{i, j}^{h 2}$. Hence, we present here the formulation for a possible match between these two utility terms.

We define sets Cond and Reb to denote the position of condenser and reboiler blocks within the superstructure. Also, Cond ${ }^{\text {hex }} \subseteq$ Cond and Reb $b^{\text {hex }} \subseteq$ Reb sets designate the condenser and reboiler blocks that are allowed to be included in the heat exchanger network. We define $h e x=\left\{i, j, i^{\prime}, j^{\prime} \mid(i, j) \in\right.$ Cond $^{\text {hex }} A N D\left(i^{\prime}, j^{\prime}\right) \in$ Reb $\left.^{\text {hex }}\right\}$ to denote the building block pairs that

are allowed to matched with each other. We define $q_{i, j, i^{\prime} j^{\prime}}^{h e x}$ to denote the amount of heat that is exchanged between condenser block $B_{i, j}$ and reboiler block $B_{i^{\prime}, j^{\prime}}$. Then, we write the following 
energy balances for a possible integration between the two blocks:

$$
\begin{gathered}
Q_{i, j}^{h 2}=Q_{i, j}^{h 3}+\sum_{\left(i^{\prime}, j^{\prime}, i, j\right) \in h e x} q_{i^{\prime} j^{\prime}, i, j}^{h e x} \quad i, j \in R e b^{h e x} \\
Q_{i, j}^{c}=Q_{i, j}^{c 3}+\sum_{\left(i, j, i^{\prime}, j^{\prime}\right) \in h e x} q_{i, j, i^{\prime}, j^{\prime}}^{h e x} \quad i, j \in \text { Cond }^{h e x}
\end{gathered}
$$

In the above constraints, if there is no heat integration between the two blocks, or there is partial heat integration, then the heat duty terms $Q_{i, j}^{h 3}$ and $Q_{i, j}^{c 3}$ terms are used to quantify these residual heat terms.

For a feasible heat exchange operation, we need to also satisfy the following approach temperature constraint assuming isothermal condenser and reboiler operation:

$$
d t_{i, j, i^{\prime}, j^{\prime}}^{h e x} \leq T_{i, j}-T_{i^{\prime}, j^{\prime}} \quad i, j, i^{\prime}, j^{\prime} \in h e x
$$

Above constraint requires that for all the condenser-reboiler block matches included in hex set, condenser block temperatures should be higher than the reboiler blocks. A minimum approach temperature of $1 \mathrm{~K}$ is used in this work, i.e. $d t_{i, j, i^{\prime}, j^{\prime}}^{\text {hex }} \geq 1$. Note that, in the example problem, we specifically investigate the effect of heat integration on the flowsheet alternative F4 and use the above constraint to realize a feasible operation. However, one can introduce binary variables to activate these constraints only if the heat exchanger is needed. These type of models can be found in our previous works. ${ }^{4,5}$

\section{Physical Parameters}

Density parameters are obtained through regression on the data taken from ASPEN Plus.

Table S2: Molar Concentration Parameters

$\begin{array}{cccc} & \rho_{k}^{d 1}\left[\frac{\mathrm{kmol}}{\mathrm{m}^{3}}\right] & \rho_{k}^{d 2}\left[\frac{\mathrm{kmol}}{\mathrm{m}^{3} \mathrm{~K}}\right] & \rho_{k}^{d 3}\left[\frac{\mathrm{kmol}}{\mathrm{m}^{3} \mathrm{~K}^{2}}\right] \\ \mathrm{W} & 75.22 & -0.06505 & 0 \\ \text { EO } & 1.655 & 0.1263 & -0.0002286 \\ \text { EG } & 23.66 & -0.01804 & 0 \\ \text { DEG } & 13.59 & -0.009986 & 0\end{array}$

Vapor pressure is calculated based on Antoine equation with the parameters obtained from Aspen Plus. 
Table S3: Antoine Equation Parameters

$\begin{array}{cccc} & A_{k}^{\text {ant }} & B_{k}^{\text {ant }} & C_{k}^{\text {ant }} \\ \text { EO } & 10.884 & 3152 & 7.667 \\ \text { W } & 11.680 & 3828 & -45.412 \\ \text { EG } & 11.963 & 4764 & -72.275 \\ \text { DEG } & 11.256 & 4655 & -103.551\end{array}$

Temperature dependence of specific enthalpy for each species is obtained through ASPEN Plus and fitted to a linear function.

Table S4: Enthalpy Parameters

$\begin{array}{ccccc} & H_{k}^{a, v a p} & H_{k}^{b, v a p} & H_{k}^{a, l i q} & H_{k}^{b, l i q} \\ \text { EO } & -74.6 & 0.0697 & -143.8 & 0.2037 \\ \text { W } & -252.3 & 0.0348 & -320.0 & 0.1068 \\ \text { EG } & -424.3 & 0.1030 & -519.0 & 0.1924 \\ \text { DEG } & -603.4 & 0.1823 & -723.0 & 0.3043\end{array}$

\section{Capital Cost Functions}

In Eqs. 16-26, objective function for a reactive separation column is provided. Here, we describe a more general form that we have used in implementing all the optimization problems. First we provide the general form of the objective functions as obtained from the literature.

For all equipment, material of construction is selected as stainless steel as in a typical ethylene glycol plant. ${ }^{6}$ Also, these costs take into account the pressure correction factors given in Douglass ${ }^{7}$ for the maximum allowed pressure within the process (i.e. $36 \mathrm{~atm}$ ).

Cost of heat exchangers are calculated based on heat exchange area: ${ }^{7}$

$$
\operatorname{Cost}^{H E X}(\$)=\frac{M \& S}{280} 101.3 A\left[f t^{2}\right]^{0.65}\left(2.29+F_{m}\left(F_{d}+F_{p}\right)\right)
$$

$F_{m}, F_{d}$ and $F_{p}$ are taken as $3.75,1$ and 0.39 , respectively. PFR cost is approximated as a multitubular heat exchanger with 1-in diameter tubes which provide fully developed turbulent flow through the reactor. ${ }^{6}$ Hence, this cost function is also used as a basis for the PFR. Reactive and non-reactive distillation column shell cost functions are taken as: ${ }^{7}$

$$
\operatorname{Cost}^{\text {Column }}(\$)=\frac{M \& S}{280} 101.9 D[f t]^{1.066} H[f t]^{0.802}\left(2.18+\left(F_{m} F_{p}\right)\right)
$$


$F_{m}$ and $F_{p}$ are taken as 2.25 and 1.60 , respectively. Column tray costs are given as: ${ }^{7}$

$$
\operatorname{Cost}^{\text {trays }}(\$)=\frac{M \& S}{280} 4.7 D[f t]^{1.55} H[f t]\left(F_{s}+F_{t}+F_{m}\right)
$$

$F_{s}, F_{t}$ and $F_{m}$ are taken as 1,0 and 1.7 , respectively. Pump costs are taken from Espatolero et al.: 8

$$
\text { Cost }^{\text {pump }}=C_{B}(W(k W) / 4)^{0.55} f_{M} f_{P} f_{T} f_{M \& S}
$$

$C_{B}, f_{M}, f_{P}, f_{T}$ and $f_{M \& S}$ are taken as $9840,1.0,1.5,1.0$ and 1.261 , respectively.

\section{Economic Objective Functions}

Here, we provide the more detailed version of the objective functions with the variables and constraints introduced above. Economic objective for maximizing ROI is implemented as follows:

$$
\max \quad R O I \times I C C \times \alpha^{T C I}=A N P
$$

Here $R O I$ is the return on investment, $I C C$ is the installed capital cost, $A N P$ is the annual net (after-tax) profit and $\alpha^{T C I}$ is the total capital investment cost multiplier to obtain the total investment cost as a function of $I C C$. ICC is the summation of all capital investment required for the individual pieces of equipment. All cost functions are calculated as given above by assuming an M\&S index of $1431.7^{9}$ and multipliers are modified for the flow rate, energy or 
volume units we used in the model.

$$
\begin{aligned}
& I C C=\sum_{e \in E^{\text {Dist }}} 64.89\left(H E_{e}\right)\left(D_{e}\right)^{1.55}+\sum_{e \in E^{D i s t}} 3011.6\left(H E_{e}\right)^{0.802}\left(D_{e}\right)^{1.066} \\
& +18612 \sum_{i, j, i^{\prime}, j^{\prime} \in L N^{p u m p}}\left(J_{i, j, i^{\prime}, j^{\prime}}^{\text {pump }} / 4\right)^{0.55}+488043 \sum_{e \in E^{P F R}}\left(V_{e}^{P F R}\right)^{0.65} \\
& +18208 \sum_{i, j \in R e b / R e b^{h e x}}\left[\frac{Q_{i, j}^{h 2}}{U^{r e b}\left(T^{H U}-T_{i, j}\right)}\right]^{0.65} \\
& +18208 \sum_{i, j \in \text { Cond } / \text { Cond }^{\text {hex }}}\left[\frac{Q_{i, j}^{c}}{U^{\text {cond }}\left(\frac{\Delta T_{i, j}^{i n, \text { cond }}+\Delta T_{i, j}^{\text {out }, \text { cond }}}{2}\right)}\right]^{0.65} \\
& +18208 \sum_{i, j \in R e b^{h e x}}\left[\frac{Q_{i, j}^{h 3}}{U^{r e b}\left(T^{H U}-T_{i, j}\right)}\right]^{0.65} \\
& +18208 \sum_{i, j \in C o n d^{h e x}}\left[\frac{Q_{i, j}^{c 3}}{U^{\text {cond }}\left(\frac{\Delta T_{i, j}^{i n, \text { cond }}+\Delta T_{i, j}^{\text {out }, \text { cond }}}{2}\right)}\right]^{0.65} \\
& +18208 \sum_{i, j \in B \text { Cool }}\left[\frac{Q_{i, j}^{c}}{U^{B C o o l}\left(\frac{\Delta T_{i, j}^{i n, c o o l}+\Delta T_{i, j}^{\text {out }, \text { cool }}}{2}\right)}\right]^{0.65} \\
& +18208 \sum_{i, j \in \text { BHeat }}\left[\frac{Q_{i, j}^{h}}{U^{B H e a t}\left(\frac{\Delta T_{i, j}^{\text {in,heat }}+\Delta T_{i, j}^{\text {out }, \text { heat }}}{2}\right)}\right]^{0.65} \\
& +18208 \sum_{i, j, i^{\prime}, j^{\prime} \in h e x}\left[\frac{q_{i, j, i^{\prime}, j^{\prime}}^{\text {hex }}}{U^{h e x} d t_{i, j, i^{\prime}, j^{\prime}}^{\text {hex }}}\right]^{0.65}
\end{aligned}
$$

Here, the first and second terms are the installed capital costs (ICC) for the distillation column trays and the column shell, respectively. $H E_{e}$ and $D_{e}$ are the total height and diameter of the columns $e$, respectively, in $\mathrm{ft}$. These are calculated based on Eqs. 23-25 as a function of the vapor flow rate, temperature, pressure and reaction volume of the individual blocks that these equipment are comprised of indicated by the set $E q R$.

The third term is the ICC for the pumps located on the jump streams and $J_{i, j, i^{\prime}, j^{\prime}}^{p u m p}$ is the work needed in $\mathrm{kW}$. The work term is calculated as follows:

$$
J_{i, j, i^{\prime}, j^{\prime}}^{\text {pump }}=\frac{10^{5} \times\left(\sum_{k} J_{i, j, i^{\prime}, j^{\prime}, k} M W_{k}\right)\left(P_{i^{\prime}, j^{\prime}}-P_{i, j}\right)}{\rho_{i, j}^{\text {mix }} \times 1000 \times 3600 \times \eta^{\text {pump }} \times \eta^{\text {motor }}}, \quad i, j, i^{\prime}, j^{\prime} \in L N^{\text {pump }}
$$


Here, $M W_{k}$ is the molecular weight of component $k, \rho_{i, j}^{\text {mix }}$ is the liquid density which is assumed to be $1000 \mathrm{~kg} / \mathrm{m}^{3}$, pressure terms are in atm, $\eta^{\text {pump }}$ and $\eta^{\text {motor }}$ are the pump and motor efficiencies which are taken as 0.5 and 0.9 , respectively.

The forth term is the ICC for the PFR as a function of the PFR volume. Volume of the PFR unit is calculated as the summation of the individual volumes of the building blocks that represent the reactor:

$$
\begin{gathered}
V_{e}^{P F R}=\sum_{i, j \in E q R} V_{i, j}, \quad e \in E^{P F R} \\
V_{i, j}=V_{i^{\prime}, j^{\prime}} \quad e \in E^{P F R}, i, j, \in E q R, i^{\prime}, j^{\prime} \in E q R \\
P_{i, j}=P_{i^{\prime}, j^{\prime}} \quad e \in E^{P F R}, i, j, \in E q R, i^{\prime}, j^{\prime} \in E q R
\end{gathered}
$$

Here, the second and third equations state that the volumes and pressures of the blocks belonging to the same PFR unit should be the same.

The fifth to eleventh terms in Eq. S50 are for the ICC of the reboilers, condensers, block heaters, block coolers and heat exchangers. All heat duty terms are in $\mathrm{MJ} / \mathrm{h}$. The overall heat transfer coefficients $U^{\text {BHeat }}$ and $U^{r e b}$ are taken as $5.11 \mathrm{MJ} / \mathrm{h} / \mathrm{m}^{2} / \mathrm{K} . U^{\text {cond }}, U^{B C o o l}$ and $U^{\text {hex }}$ are taken as $8.176 \mathrm{MJ} / \mathrm{h} / \mathrm{m}^{2} / \mathrm{K}$. Specifically, the fifth and sixth terms are for the reboiler and condenser blocks that are not allowed to exchange heat with the other blocks, respectively. Here, $T^{H U}$ is the hot utility temperature, i.e. $527.2 \mathrm{~K}$. For the condensers, temperature driving force is assumed to be the linear average between the inlet and outlet temperature differences. These terms are calculated as follows:

$$
\begin{gathered}
\Delta T_{i, j}^{i n, \text { cond }}=T_{i, j}-T^{C U, i n}, \quad i, j \in \text { Cond } \\
\Delta T_{i, j}^{\text {out }, \text { cond }}=T_{i, j}-T^{C U, \text { out }}, \quad i, j \in \text { Cond }
\end{gathered}
$$

$T^{C U, \text { in }}$ and $T^{C U, \text { out }}$ are the inlet and outlet temperatures for the cooling water and taken as 305.2 and $325.2 \mathrm{~K}$, respectively.

The seventh and eighth terms are the ICC for the reboilers and condensers for the residual heat required, if any, after heat integration, respectively. The ninth and tenth terms are for the block coolers and heaters, respectively. Temperature difference terms are calculated as follows:

$$
\Delta T_{i, j}^{i n, \text { cool }}=T_{i, j}-T^{C U, i n}, \quad i, j \in B \text { cool }
$$




$$
\begin{gathered}
\Delta T_{i, j}^{\text {out }, \text { cool }}=T_{i^{\prime}, j^{\prime}}-T^{C U, \text { out }}, \quad i, j, i^{\prime}, j^{\prime} \in \text { Bcoolmatch } \\
\Delta T_{i, j}^{\text {in,heat }}=T^{H U}-T_{i, j}, \quad i, j \in \text { Bheat } \\
\Delta T_{i, j}^{\text {out,heat }}=T^{H U}-T_{i^{\prime}, j^{\prime}}, \quad i, j, i^{\prime}, j^{\prime} \in \text { Bheatmatch }
\end{gathered}
$$

Here, Bcool and Bheat sets designate the block positions $B_{i, j}$ where coolers and heaters are positioned, respectively. The block temperatures, i.e. $T_{i, j}$, where these heaters/coolers are located correspond to the outlet temperatures of these units. We do not introduce any inlet temperature term for these blocks, instead we designate the inlet temperatures by specifying the source blocks for the incoming streams. These are denoted by sets Bcoolmatch and Bheatmatch for the coolers and heaters, respectively. These sets denote the position of the source block $B_{i^{\prime}, j^{\prime}}$ that we use as the inlet temperature for the block heaters/coolers positioned in block $B_{i, j}$. The final term is the ICC for the heat exchangers.

Annual net (after-tax) profit, $A N P$, can be described as:

$$
A N P=(\text { Income }-(\text { AOC }+ \text { Maintenance }+ \text { Depreciation })) \times(1-\theta)+\text { Depreciation }
$$

where AOC describes the annual operating cost. Accordingly, we calculate $A N P$ as shown below:

$$
\begin{aligned}
& A N P=\left\{n^{o p} \times\left(\sum_{i, j, k, p s \in \operatorname{Prod} B} S P_{k, p} P_{i, j, k, p}\right)-n^{o p} \times\left(\sum_{i, j, k, f \in F B} U F_{f} M_{i, j, k, f}\right)\right. \\
& -n^{o p} \times U F^{h o t}\left(\sum_{i, j \in R e b / R e b^{h e x}} Q_{i, j}^{h 2}+\sum_{i, j \in R e b^{h e x}} Q_{i, j}^{h 3}+\sum_{i, j \in B H e a t} Q_{i, j}^{h}\right) \\
& -n^{o p} \times U F^{c o l d}\left(\sum_{i, j \in \text { Cond } / \text { Cond }}^{h e x} Q_{i, j}^{c}+\sum_{i, j \in C o n d^{h e x}} Q_{i, j}^{c 3}+\sum_{i, j \in B C o o l} Q_{i, j}^{c}\right) \\
& -n^{o p} \times U F^{E l}\left(\sum_{i, j, i^{\prime}, j^{\prime} \in L N} J_{i, j, i^{\prime}, j^{\prime}}^{\text {pump }} \times 3600\right) \\
& \left.-\left(\alpha^{\text {Maint }} \times I C C \times \alpha^{F C I}\right)-\left(\alpha^{\text {Dep }} \times I C C \times \alpha^{F C I}\right)\right\}\{1-\theta\}+\left\{\alpha^{D e p} \times I C C \times \alpha^{F C I}\right\}
\end{aligned}
$$

$n^{o p}$ is the operating time and taken as 8000 hours. The first term is the revenue from the product streams. The second term is the cost of the raw materials. The third and forth terms describe 
the hot and cold utility costs, respectively. $U F^{\text {hot }}$ is taken as $0.009906 \$ /$ MJ. UF $F^{\text {cold }}$ is taken as $0.000324 \$ / M J$. The fifth term is the total cost of electricity consumption and $U F^{E l}$ is taken as $1.94 \times 10^{-7} \$ / \mathrm{kJ}$. The sixth term describes the yearly maintenance cost as a function of fixed capital investment cost. $\alpha^{F C I}$ is the fixed capital investment cost multiplier to obtain the total investment cost as a function of $I C C . \alpha^{F C I}$ is taken as 1.924. Maintenance cost is assumed as $7.5 \%$ of the fixed capital investment, i.e. $\alpha^{\text {Maint }}=0.075$. The seventh term is the depreciation cost and 10 year linear depreciation scheme is adopted. Hence, $\alpha^{D e p}=0.10$. Seventh term includes the tax rate for which the rate is assumed to be $40 \%$, i.e. $\theta=0.40$.

\section{Block Superstructure Representations for the Base Case Designs}

In Figure S1a, non-intensified flowsheet alternative is shown. Here, the first column is used to represent the plug flow reactor. 50 building blocks (shown in yellow) in series is used with the homogeneous reaction model provided by Altıokka and Karayalçın (2009) ${ }^{1}$ to obtain a 50-CSTRs-in-series model. Here, block $B_{1,1}$ acts as a reactor inlet mixer and fresh feed and recycle streams from the separator are mixed here. Block $B_{2,1}$ is used as a reactor inlet heater or cooler. Similarly, block $B_{53,1}$ is used for reactor outlet heating/cooling. This block also serves a splitter block from which jump streams are used to transport the reactor outlet to the distillation region of the block superstructure. Distillation region is comprised of blocks between blocks $B_{1,2}$ to $B_{50,3}$. Here the blocks in the second column $(j=2)$ are in liquid phase and blocks in the third column $(j=3)$ are in vapor phase. Blocks $B_{1,2}$ and $B_{1,3}$ represents the phase change from vapor to liquid in the total condenser. Similarly, blocks $B_{50,2}$ and $B_{50,3}$ are used to represent the partial reboiler. Boundaries between second and third columns except the one in the first row are designated as semi-restricted vapor-liquid phase contact boundaries to represent the phase equilibrium. The boundary in the first row is unrestricted as there is no composition change during the total condensation of the top vapor stream. Condensed vapor at $B_{1,2}$ can be either recycled to the reactor inlet mixer block $B_{1,1}$, sent to block $B_{2,2}$ as reflux stream and/or taken out as the distillate product. The bottom product from the distillation region is taken out from block $B_{50,2}$. Note that jump streams from reactor outlet block $\left(B_{53,1}\right)$ can be connected to each of the liquid phase blocks within the distillation region. the flow rate of each of these jump streams are variables by which the feed location is determined. The number of active building block 
pairs within the distillation region is a decision variable which stands for the optimal number of stages. An upper bound of 50 is set for the number of building block pairs that can be used in separation including condenser and reboiler stages.

Building block representation for the intensified reactive distillation is shown in Figure S1b. Here, representation is similar to the non-intensified flowsheet representation except that reaction is allowed to occur in the liquid phase blocks within the distillation region. Also, external feed streams can enter into the process from any of the liquid blocks.
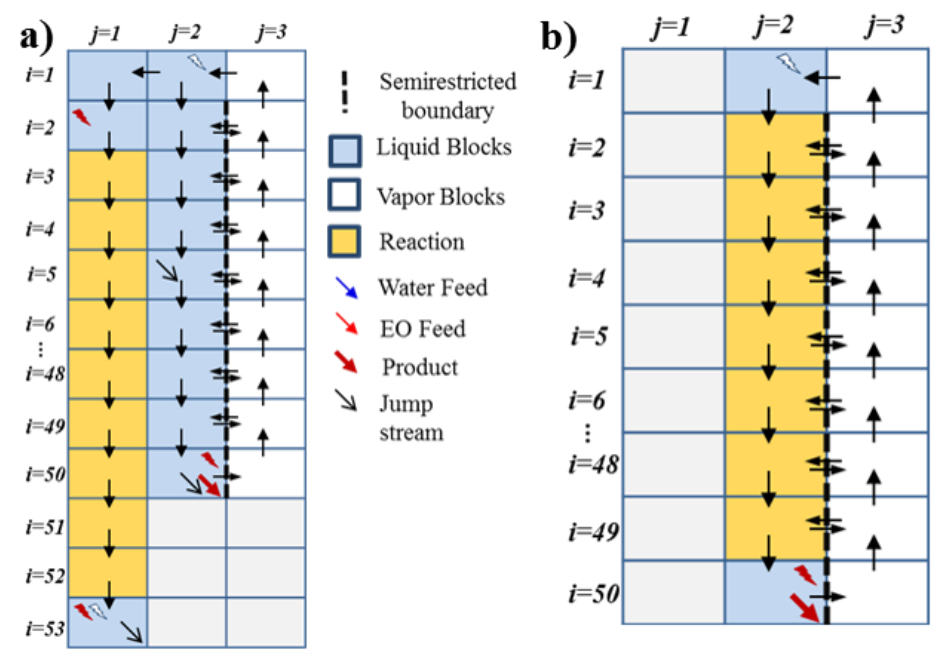

Figure S1: Building superstructure representation for a) reactor-separator-recycle system (F1), and b) intensified reactive distillation process (F2).

As the pressures of the equipment are among the optimization variables, phase of the several blocks need to be checked to fascilitate a physically realizable operation. For F1, this is done for the PFR inlet and outlet heater/cooler blocks. Also, to fascilitate liquid phase reaction, phase of the last block representing the PFR reactor is also ensured to stay in liquid phase. This is done only for the last block as the reaction is exothermic and the temperature of the reactor will always tend to increase and it will be the highest at this block where the likelihood of phase transition be the highest.

\section{Modeling Assumptions and Design Bounds}

In optimizing base-case design alternatives, following assumptions are made. Temperature and pressure bounds of the process are taken as 326-524 $\mathrm{K}$ and $0.1-36$ bar. ${ }^{10}$ Ideal vapor-liquid 
phase equilibrium is assumed. Enthalpy and liquid densities are assumed to be a function of temperature. Cost of PFR is approximated as a HEX with 1-in diameter tubes. Maximum volume of the PFR is $30 \mathrm{~m}^{3}$. Both reactive and non-reactive tray efficiencies are taken as 1 . Cost of realizing vacuum conditions is assumed to be negligible. A tray spacing of $0.61 \mathrm{~m}$ is assumed for all stages. Downcomer cross-sectional area is assumed to be $12 \%$ of the overall column cross-sectional area to prevent donwcomer flooding. F-value which is related to the flooding velocity within the column is assumed as $1.84 .^{7}$ We assumed a maximum holdup of $3 \mathrm{~m}^{3}$ on each reactive tray. Pressure drop through the $\mathrm{V}$-L building block pairs and in PFR is neglected. External liquid feed streams are assumed to be available at the required process pressure. Capital cost functions of all units include the pressure correction factors according to the maximum pressure allowed within the process (i.e. $36 \mathrm{~atm}$ ). No reaction is assumed to occur in the reboiler and condensers. Reactive tray heights are determined based on the diameter of the column, tray spacing and the additional holdup required for reaction. Maximum holdup is limited by the weir height which can be larger than the normal distillation columns. Weir heights of even $1 \mathrm{~m}$ is reported for reactive distillation systems. ${ }^{11}$ We assumed a maximum weir height of $0.5 \mathrm{~m}$. Height of the columns are restricted to $175 \mathrm{ft}$ and a maximum of L/D (Height/Diameter) ratio of 30 is assumed to prevent very tall and skinny columns. ${ }^{12}$

\section{Single Objective Optimization Results for the Base-case designs}

Here, we present the single objective optimization results for the base-case designs with change in number of stages in $\mathrm{DC}$ (for F1) and RD (for F2). We solve the single objective optimization problems for increasing building block superstructure sizes as NLPs. Results are shown in Figure S2. For F1, max ROI of $52 \%$ occurs when the number of stages for the distillation column is 7 . For F2, max ROI of 35\% occurs with 19 stages. For $\mathrm{CO}_{2}$-eq minimization results, as discussed in Section 2, we observe a monotonic decrease in emissions with the increase in number of blocks. As the reaction and separation operations are combined, the effect of number of stages is more dominant for the intensified flowsheet F2. F1 shows a rather flat profile after 12 stages. For both flowsheets we designate the flowsheet with the minimum emissions by observing the relative decrease in the emissions. We say the emissions are minimum when $\theta$ is $0.05 \%$. This corresponds to a flowsheet with 10 stages for F1 and 44 stages for F2. These designs correspond 
to the extreme points in the pareto space.

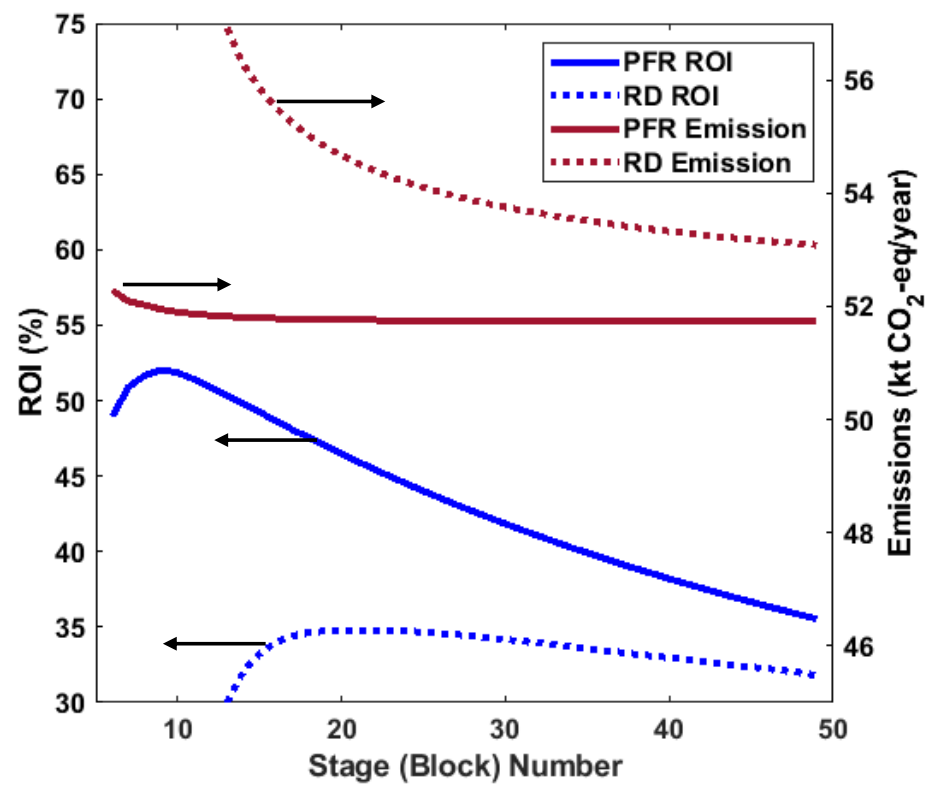

Figure S2: Optimal base case designs with single objective optimization with change in number of stages in DC (for F1) and RD (for F2). Each stage, an NLP problem is solved with both ROI and indirect emission objectives. Two red lines on top correspond to the optimization with emission objective and two blue lines at the bottom correspond to the optimization results with economic objective.

\section{Multi-Objective Optimization Results for the Base-case designs}

Here, we provide more details on the pareto solutions of the base-case designs. We observe stark changes in the optimal solutions for both F1 and F2 at certain emission levels.

For the non-intensified flowsheet F1, before the value of $53.2 \mathrm{kt} /$ year of $\mathrm{CO}_{2}$ emission, distillation pressure increases as the emission level decreases (Figure S3a). This also increases the condenser and recycle temperatures (Figure S3b). An increase in pressure results in an increase in reboiler duty (Figure S3c). Beyond the $53.2 \mathrm{kton} /$ year threshold, reduction in emissions is facilitated by the decrease in the hot utility requirement for the PFR inlet heater. However, further reduction in emission requires a decrease in the emissions from reboiler as well. To enable this, reactor volume starts to increase rapidly which also increases the conversion (Figure S3d-e). Increased conversion relieves the burden on separation by decreasing the amount of PFR 
product that needs to be separated. This also enables the PFR to operate at lower temperatures. Hence, PFR inlet heater can be eliminated. However, these changes in the flowsheet structure results in much higher capital cost requirements. Capital cost contribution from the reaction task (PFR and inlet heater) starts to exceed the cost required for the separation task (Separation column with condensers and reboilers) (Figure S3f).

a) Distillation Pressure

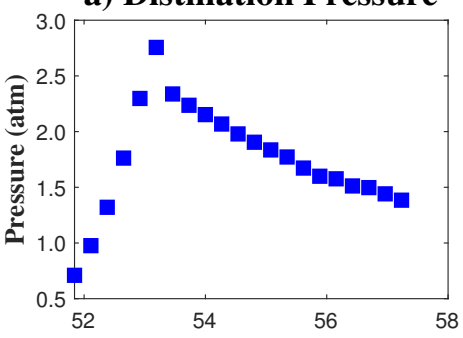

d) PFR Volume

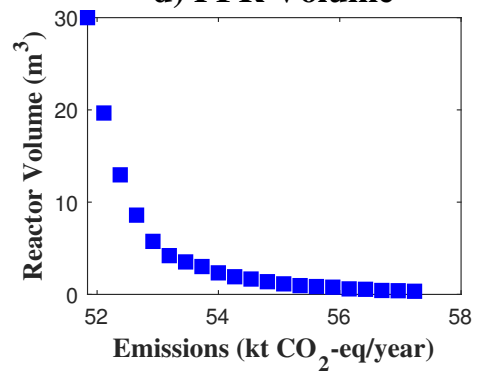

b) Distillation Temperature

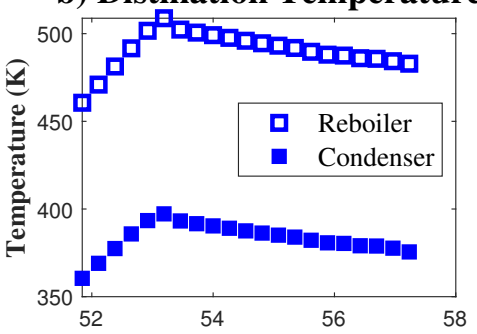

e) Single-Pass Conversion

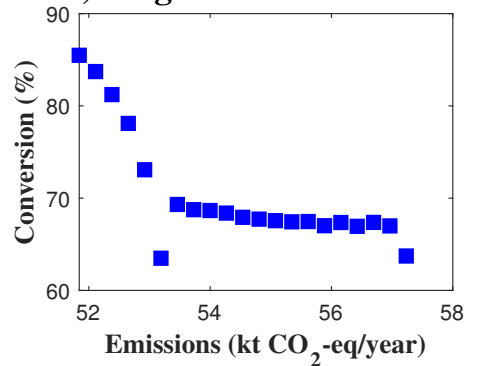

c) Hot Utility

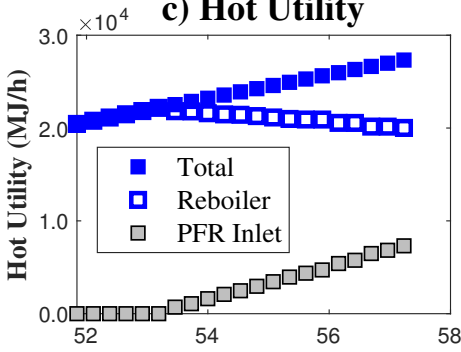

f) Capital Costs

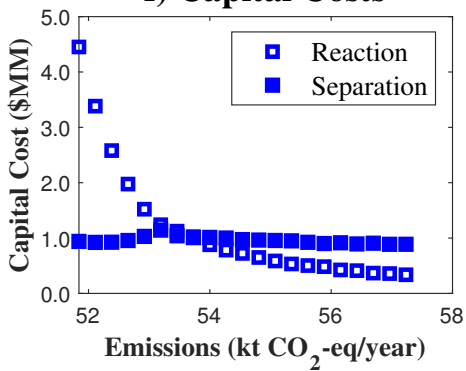

Figure S3: a) Distillation column pressure, b) distillation column reboiler and condenser temperatures, c) hot utility consumption, d) PFR volume, e) single-pass conversion, f) capital costs vs. emissions for the flowsheet F1.

For the intensified design F2, there is also an inflection point where there is a stark change in the operating conditions. While column operates at nearly same pressure levels in the higher emission levels, it starts to decrease when the emissions fall below 53.7 kton/year $\mathrm{CO}_{2}$ (Figure S4a). We also observe a change in the slope of increase in reaction volume after this point (Figure S4b). Boilup ratio of the RD also starts to decrease with a higher rate (Figure S4c). This decrease in column pressure results in lower temperatures along the reactive region and column as a whole (Figure S4e). Liquid molar holdup increases sharply to compensate for the decreasing temperatures along the reactive region. We also observe the effect of these changes on the capital cost. Column cost (summation of tray and shell costs) starts to increase sharply after this point while reboiler cost decreases due to the decreased pressure. This is similar in nature 
to the flowsheet F1 in which going towards lower emissions requires to increase the reactor cost for decreasing the separation cost. Yet, unlike the non-intensified case, RD cannot manipulate the conditions to that extent to increase the relative volatility and ease the separation as they are coupled in the intensified design.
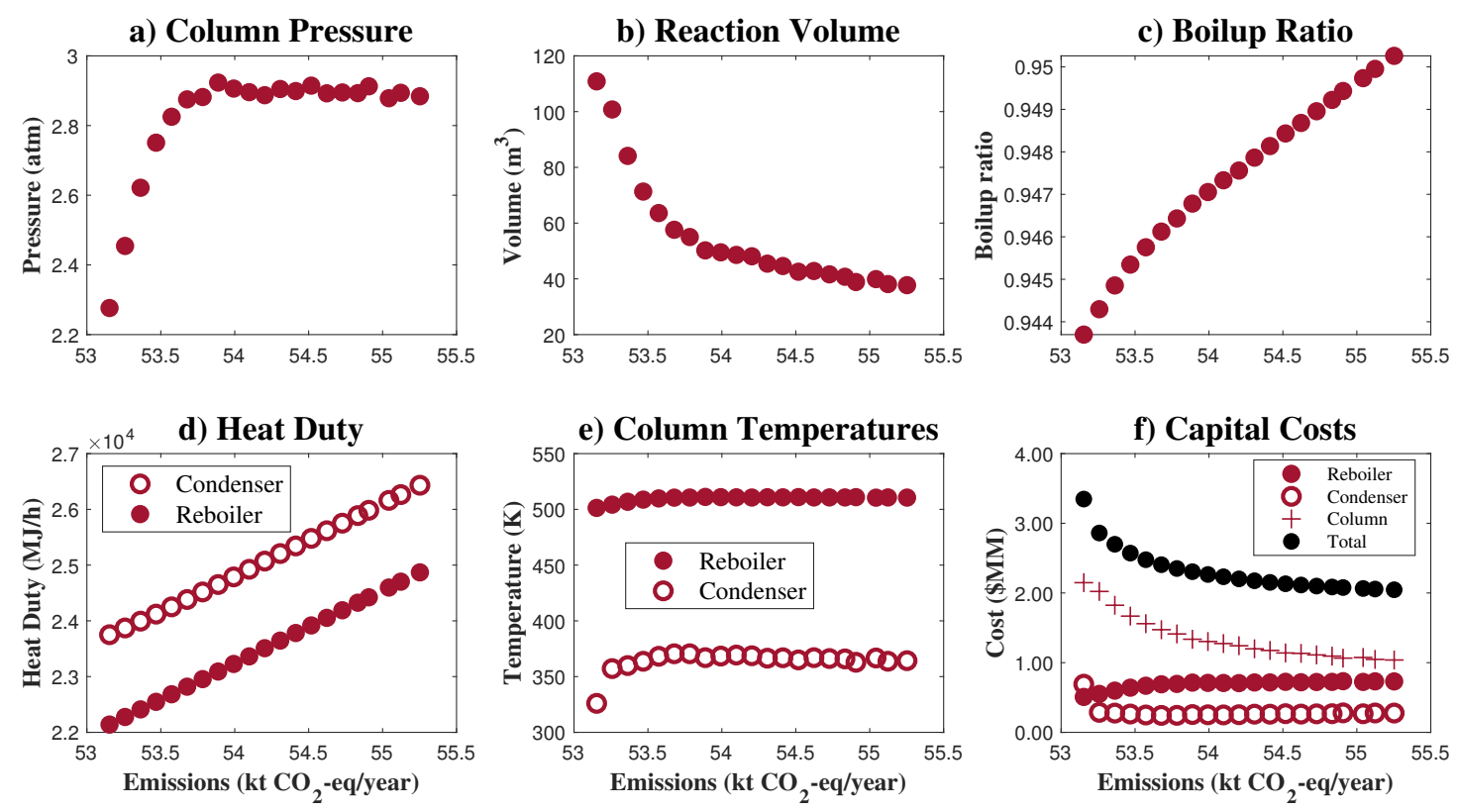

Figure S4: a) Column pressure, b) total reaction volume, c) boilup ratio, d) condenser and reboiler heat duties, e) reboiler and condenser temperatures, and f) capital costs vs. emissions for the flowsheet F2.

\section{Cost breakdown of the optimal solutions}

Here, a detailed cost breakdown for the optimal base-case flowsheets as well as the generated designs are provided. Note that results correspond to the ultimate optimal solutions obtained considering larger superstructures. 
Table S5: Cost breakdown for the base case designs.

\begin{tabular}{|c|c|c|c|c|}
\hline Flowsheet & $\mathrm{F} 1$ & $\mathrm{~F} 1$ & $\mathrm{~F} 2$ & $\mathrm{~F} 2$ \\
\hline Objective & $\max \mathrm{ROI}$ & $\min \mathrm{CO}_{2}$ & $\max \mathrm{ROI}$ & $\min \mathrm{CO}_{2}$ \\
\hline Stage & 7 & 10 & 19 & 44 \\
\hline Height (m) & 7.3 & 9.1 & 21.6 & 48.3 \\
\hline Diameter (m) & 1.9 & 2.2 & 2.6 & 2.8 \\
\hline Rxn Volume $\left(\mathrm{m}^{3}\right)$ & 0.3 & 30 & 37.7 & 110.9 \\
\hline \multicolumn{5}{|c|}{ Costs } \\
\hline Shell Cost $(\$ 1000)$ & 269 & 376 & 909 & 1835 \\
\hline Tray Cost $(\$ 1000)$ & 26.2 & 41.2 & 129 & 313 \\
\hline Reboiler $(\$ 1000)$ & 335 & 261 & 731 & 508 \\
\hline Condenser $(\$ 1000)$ & 257 & 261 & 277 & 691 \\
\hline Reactor $(\$ 1000)$ & 243 & 4452 & - & - \\
\hline Heater $(\$ 1000)$ & 94 & 0 & - & - \\
\hline Pump (\$1000) & 42.6 & 8.6 & - & - \\
\hline Total Installed Capital (\$1000) & 1267 & 5400 & 2046 & 3347 \\
\hline Hot Utility ( $\$ 1000 /$ year) & 2165 & 1620 & 1971 & 1755 \\
\hline Cold Utility ( $\$ 1000 /$ year $)$ & 75 & 58 & 69 & 62 \\
\hline Fresh EO (\$1000/year) & 6473 & 6474 & 6472 & 6474 \\
\hline Fresh W (\$1000/year) & 0.42 & 0.42 & 0.42 & 0.42 \\
\hline Electricity ( $\$ 1000 /$ year $)$ & 10 & 0.6 & - & - \\
\hline Op. Cost $(\$ 1000 /$ year $)$ & 8724 & 8153 & 8512 & 8291 \\
\hline \multicolumn{5}{|c|}{ Emissions } \\
\hline Steam (kt $\mathrm{CO}_{2} /$ year $)$ & 21.14 & 15.81 & 19.24 & 17.13 \\
\hline $\mathrm{EO}(\mathrm{kt} \mathrm{CO} 2 /$ year $)$ & 36.02 & 36.02 & 36.01 & 36.03 \\
\hline Electricity (kt $\mathrm{CO}_{2} /$ year) & 0.08 & 0.004 & - & - \\
\hline Total (kt $\mathrm{CO}_{2} /$ year) & 57.23 & 51.84 & 55.25 & 53.15 \\
\hline \multicolumn{5}{|c|}{ Economics } \\
\hline Total Annualized Cost $(\$ 1000 /$ year $)$ & 9150 & 9971 & 9201 & 9418 \\
\hline Annual Economic Prof. ( $\$ 1000 /$ year) & 1493 & 1796 & 1612 & 1732 \\
\hline Total Capital Investment $(\$ 1000)$ & 2868 & 12223 & 4631 & 7576 \\
\hline ROI (\%/year) & 52 & 15 & 35 & 23 \\
\hline
\end{tabular}


Table S6: Cost breakdown for the generated designs.

\begin{tabular}{|c|c|c|c|c|c|}
\hline Flowsheet & F3 & F4 & $\mathrm{F} 4$ & F5 & F5 \\
\hline Objective & $\max \mathrm{ROI}$ & $\max \mathrm{ROI}$ & $\min \mathrm{CO}_{2}$ & $\max \mathrm{ROI}$ & $\min \mathrm{CO}_{2}$ \\
\hline Stage RD & 23 & 16 & 28 & 21 & 28 \\
\hline Stage DC & - & 4 & 10 & 5 & 10 \\
\hline Height RD (m) & 24.9 & 16.6 & 24.7 & 20.0 & 34.1 \\
\hline Height DC (m) & - & 5.5 & 9.1 & 6.1 & 9.1 \\
\hline Diameter RD (m) & 1.8 & 0.6 & 2.8 & 0.8 & 2.8 \\
\hline Diameter DC (m) & - & 2.0 & 2.5 & 1.7 & 2.2 \\
\hline Reaction Volume $\left(\mathrm{m}^{3}\right)$ & 19.8 & 0.9 & 27.3 & 2.0 & 84.0 \\
\hline \multicolumn{6}{|c|}{ Costs } \\
\hline Shell Cost RD (\$1000) & 681 & 142 & 1069 & 233 & 1387 \\
\hline Shell Cost DC ( $\$ 1000)$ & - & 225 & 432 & 204 & 371 \\
\hline Tray Cost RD (\$1000) & 83 & 9 & 160 & 18 & 221 \\
\hline Tray Cost DC (\$1000) & - & 21 & 50 & 18 & 40 \\
\hline Condenser RD ( $\$ 1000)$ & 251 & 0 & 0 & 0 & 0 \\
\hline Condenser DC ( $\$ 1000)$ & - & 252 & 647 & 232 & 467 \\
\hline Reboiler RD (\$1000) & 445 & 258 & 130 & 262 & 129 \\
\hline Reboiler DC (\$1000) & - & 94 & 297 & 0 & 0 \\
\hline $\operatorname{HEX}(\$ 1000)$ & - & - & - & 193 & 1852 \\
\hline Pump $(\$ 1000)$ & - & 26 & 38 & 24 & 10 \\
\hline Total Ins. Cap. $(\$ 1000)$ & 1459 & 1027 & 2823 & 1183 & 4478 \\
\hline Hot Utility ( $\$ 1000 /$ year) & 2063 & 1789 & 1536 & 1045 & 867 \\
\hline Cold Utility ( $\$ 1000 /$ year) & 71 & 63 & 56 & 39 & 34 \\
\hline Fresh EO (\$1000/year) & 6461 & 6465 & 6475 & 6467 & 6475 \\
\hline Fresh W (\$1000/year) & 0.42 & 0.42 & 0.42 & 0.67 & 0.42 \\
\hline Electricity ( $\$ 1000 /$ year $)$ & - & 4.0 & 8.3 & 3.5 & 0.7 \\
\hline Op. Cost ( $\$ 1000 /$ year $)$ & 8595 & 8322 & 8074 & 7556 & 7377 \\
\hline \multicolumn{6}{|c|}{ Emissions } \\
\hline Steam (kt $\mathrm{CO}_{2} /$ year) & 20.13 & 17.47 & 14.99 & 10.20 & 8.46 \\
\hline $\mathrm{EO}(\mathrm{kt} \mathrm{CO} /$ year $)$ & 35.95 & 35.98 & 36.03 & 35.99 & 36.03 \\
\hline Electricity (kt $\mathrm{CO}_{2} /$ year) & 0 & 0.03 & 0.07 & 0.03 & 0.01 \\
\hline Total (kt $\mathrm{CO}_{2} /$ year) & 56.09 & 53.47 & 51.08 & 46.22 & 44.50 \\
\hline \multicolumn{6}{|c|}{ Economics } \\
\hline Total Annual. Cost (\$1000/year) & 9086 & 8668 & 9025 & 7954 & 8884 \\
\hline Annual Ec. Prof. ( $\$ 1000 /$ year) & 1568 & 1736 & 1867 & 2194 & 2270 \\
\hline Total Cap. Inv. (\$1000) & 3303 & 2325 & 6390 & 2678 & 10135 \\
\hline ROI (\%/year) & 48 & 75 & 29 & 82 & 22 \\
\hline
\end{tabular}




\section{References}

1. Altiokka, M. R.; Akyalcin, S. Kinetics of the hydration of ethylene oxide in the presence of heterogeneous catalyst. Industrial \& Engineering Chemistry Research 2009, 48, 10840-10844.

2. Demirel, S. E.; Li, J.; Hasan, M. M. F. Systematic process intensification using building blocks. Computers \& Chemical Engineering 2017, 105, 2-38.

3. Demirel, S. E.; Li, J.; Hasan, M. F. Simultaneous process synthesis and heat integration using a single superstructure. Computing and Systems Technology Division 2017 - Core Programming Area at the 2017 AIChE Annual Meeting 2017, 2017-October, 199-200.

4. Demirel, S. E.; Li, J.; Hasan, M. F. A general framework for process synthesis, integration, and intensification. Industrial \&5 Engineering Chemistry Research 2019, 58, 5950-5967.

5. Li, J.; Demirel, S. E.; Hasan, M. M. F. Process Integration Using Block Superstructure. Industrial \& Engineering Chemistry Research 2018, 57, 4377-4398.

6. Dye, R. F. Ethylene glycols technology. Korean Journal of Chemical Engineering 2001, 18, $571-579$.

7. Douglas, J. M. Conceptual design of chemical processes; McGraw-Hill New York, 1988; Vol. 1110.

8. Espatolero, S.; Romeo, L. M.; Cortés, C. Efficiency improvement strategies for the feedwater heaters network designing in supercritical coal-fired power plants. Applied Thermal Engineering 2014, 73, 449-460.

9. Zhang, Q.; Liu, M.; Zeng, A. Performance enhancement of pressure-swing distillation process by the combined use of vapor recompression and thermal integration. Computers $\&$ Chemical Engineering 2019, 120, 30-45.

10. Barecka, M. H.; Skiborowski, M.; Górak, A. Practical Aspects of Chemical Engineering; Springer, 2018; pp 17-34.

11. Hasselbach, H. J.; Koerfer, M.; Gruener, C. P.; Hanrath, F. H.; Stock, J.; Gangadwala, J.; Krull, H. Method of production of a methionine salt. 2015; US Patent 9,023,284. 
12. Douglas, J. A hierarchical decision procedure for process synthesis. AIChE Journal 1985, 31, 353-362. 